Published in final edited form as:

Wiley Interdiscip Rev Cogn Sci. 2012 ; 3(3): . doi:10.1002/wcs.1175.

\title{
Recent progress in perceptual learning research
}

\author{
Yuka Sasaki, \\ Athinoula A. Martinos Center for Biomedical Imaging, Department of Radiology, Massachusetts \\ General Hospital, Harvard Medical School \\ José E. Náñez Sr., and \\ Division of Social and Behavioral Sciences, New College of Interdisciplinary Arts and Sciences, \\ Arizona State University \\ Takeo Watanabe \\ Department of Psychology and Center for Neuroscience, Boston University
}

\section{Abstract}

Perceptual learning is defined as long-term improvement in perceptual or sensory systems resulting from repeated practice or experience. As the number of perceptual learning studies has increased, controversies and questions have arisen regarding divergent aspects of perceptual learning, including: (1) stages in which perceptual learning occurs, (2) effects of training type, (3) changes in neural processing during the time course of learning, (4) effects of feedback as to correctness of a subject's responses and (5) double-training. Here we review each of these aspects and suggest fruitful directions for future perceptual learning research.

Our sensory and perceptual systems change according to environments we experience regularly. While most of the changes occur very early, during fixed periods of development, known as critical periods ${ }^{1-3}$, adult sensory and perceptual systems remain sufficiently plastic to adjust to some important environmental changes ${ }^{4-8}$. For example, in adults, training or exposure to a sensory feature leads to higher sensitivity or performance on the feature that can be retained for months or years 9,10 . Such changes are known as perceptual learning, which has been shown to occur in all sensory modalities other than vision, that is, in auditory ${ }^{11}$, olfactory ${ }^{12}$, taste ${ }^{13}$, and tactile ${ }^{14}$. Perceptual learning is usually distinguished from categorization learning (but see ${ }^{15}$ ). In this review, we focus on perceptual learning in vision.

A large number of psychophysical studies have shown that perceptual learning is highly specific for location of visual stimulus presentation in the visual field. That is, perceptual learning leads to higher performance on the task if the stimulus is subsequently presented in the location where the task has been previously trained, but not in an untrained location where the stimulus has not been presented (to name a few studies) ${ }^{7-9,16-30}$, although it may depend on task difficulty ${ }^{31}$ or double training ${ }^{32}$. Perceptual learning specificity has been demonstrated for features such as stimulus orientation. For example, visual stimulus presentation at one orientation, say vertical, does not transfer to the orthogonal, or horizontal in this case, orientation that forms a right angle ${ }^{8,23,33}$ and motion direction ${ }^{9,34-37}$. Moreover, perceputual learning is trained-eye specific, that is, perceptual learning of a stimulus as a result of presentation to one eye is reduced or eliminated if the stimulus is presented to the other eye ${ }^{17,38,39}$, although it has been suggested that eye specificity also depends on the mechanism ${ }^{40}$ and time passage ${ }^{17}$ or can be changed in unclear 
parameters ${ }^{41}$. The high specificity nature of perceptual learning appeared to be evidence that perceptual learning involves early visual stage based on results of the neurophysiological studies below: First, neurons in the early visual cortex tend to possess smaller receptive fields than those in higher areas ${ }^{42}$. Second, specificity of some primitive features such as orientation and motion direction tends to be higher in cells in lower-level than higher-level visual processing areas ${ }^{43}$.

However, the results of high specificity have been re-examined and lead to further facets, which we will review in this article. We will address following issues recently debated intensively: (1) what are the visual processing stages in which perceptual learning occurs, (2) what are the effects of differences in types of visual training, (3) what changes occur in neural processing during the real- or near-real-time course of learning and (4) what are the effects of feedback regarding correct or incorrect subject responses? Additionally, another set of new findings emerges: (5) effects of so called double training on transfer for position and other factors. Research findings to date regarding these questions are presented below. However, our focus will be limited to perceptual learning of primitive visual features. For those who would be interested in more expanded issues in perceptual learning, we would recommend to read further articles by us ${ }^{44}$ and others 45,46 .

\section{What are the visual processing stages in which perceptual learning occurs?}

It has been suggested that high specificity for location and features, and lack of feedback requirement (see below for detail) constitute evidence for involvement of low-level stages including the primary visual processing cortex (V1). The perceptual learning may involve changes in the stage of sensory representation, such as in V1 $24,47-53$.

However, some researchers have suggested other possibilities. For example, Petrov et al. (2005) built a model in which location and feature specificity can be explained by referring to higher level(s) of visual processing ${ }^{54}$. Specifically, they described perceptual learning as a result of changes in task-specific selective 'weighting' (i.e., the strength of neural connections) between low-level visual stages and a higher stage at which decision concerning how visual signals are interpreted to guide responses (decision stage) occurs. Some neurophysiological studies support the Petrov et al model. For example, a single-unit recording experiment found that cell responses in V4 of monkeys changed as a result of perceptual learning, while responses were not observed in $\mathrm{V} 1$ cell recordings ${ }^{55}$, thus, casting a doubt on that the perceptual learning involves the early stage of visual processing. This finding is also in accord with Raiguel et al. study 56 in that V4 neurons are likely to involve the orientation discrimination task. The Yang et al. study is at odds with the changes in V1 associated with perceptual learning ${ }^{55}$. Another single-unit recording study indicated that perceptual learning of motion accompanies changes in the lateral intraparietal area ${ }^{57}$, which is known to represent the transformation of visual motion signals into responses by saccadic eye movements, namely, to represent a perceptual decision ${ }^{58}$. Interestingly, they did not find changes in MT neurons, which represent the direction of motion ${ }^{57}$. Again this report does not support changes in the sensory representation underlying the perceptual learning, although the experiment did not test areas lower than MT

Previous studies looked into the neural changes that are correlated with improvement in a perceptual learning task. Instead of taking a correlational approach, we developed a causeand-effect approach, and investigated whether the adult early visual cortex is sufficient to cause perceptual learning ${ }^{59}$. In this study, we installed brain patterns only in the early visual cortex, patterns that correspond to an orientation, without actual stimulus presentation or participants' awareness of what they have to learn, by means of an online-feedback method 
that uses decoded functional magnetic resonance imaging (fMRI) signals. The repetitive installation of the brain patterns resulted in an increased sensitivity to that particular orientation. The study strongly suggests that the early visual cortex is so plastic that mere induction of activity pattern is sufficient to cause perceptual learning at least in the case of an orientation discrimination task.

\section{What are the effects of differences in types of visual training?}

Task training type can be divided into two categories, task-relevant learning (learning of a sensory feature to which attention is directed during training) and task-irrelevant learning (learning of a feature to which attention is not directed during training).

It has been controversial whether exposure-based perceptual learning is possible. Some studies favored the view that exposure-based perceptual learning is impossible, and attention to a visual feature during training is necessary for learning the feature. Specifically, while performance enhancement occurred on the feature subjects worked on (task-relevant feature), no significant performance enhancement on task-irrelevant features presented during training on a task-relevant feature was observed $25,26,31,60$.

On the other hand, there is the issue that important items or features are repeatedly presented in a natural environment. Thus, it may not be unreasonable to assume that perceptual learning can in fact occur when features are merely presented repeatedly without directed attention. The finding that feedback is not required for perceptual learning ${ }^{23,} 61$ led some researchers to suggest that perceptual learning occurs mainly on the basis of exposuredependent rules, in other words, mere exposure to a stimulus is sufficient to produce perceptual learning $23,36,61-64$.

Research by Takeo Watanabe, José E. Náñez, Sr. and Yuka Sasaki (2001) identified a new type of perceptual learning in which visual plasticity occurs without the subject directing attention to the feature to be learned, that is, task-irrelevant learning ${ }^{65}$. Specifically, Watanabe, Náñez, and Sasaki conducted a series of experiments in which subjects were repeatedly exposed to a background that consisted of a small number of coherently moving dots (dot motion in the same direction and speed) and a much larger number of randomly moving dots. Despite being below the subjects' perceptual threshold (and therefore, invisible) and irrelevant to the subjects' central task, repetitive exposure to a constant direction of a subthreshold coherent motion display improved performance. This type of learning is known as task-irrelevant perceptual learning (TIPL) as opposed to task-relevant perceptual learning.

More recently, TIPL was reported to occur as the result of temporal-pairing of their subthreshold, task-irrelevant, motion display and a task-target in the central task (i.e., concurrent presentation of the task-irrelevant and task-relevant stimuli) (Seitz \& Watanabe, 2003). Under such stimuli conditions, learning or improved sensitivity was observed only for the motion-direction temporally paired with the task-target, but not for motion-directions paired with non-target items. These results demonstrate that while attention to a taskirrelevant feature is not necessary for the feature to be learned, temporal engagement (pairing) with some central feature is necessary for the task-irrelevant feature to be learned.

Aaron Seitz and Takeo Watanabe ${ }^{66}$ have proposed a Unified model of perceptual learning that attempts to explain task-relevant and task-irrelevant learning of the type described above. In this model, perceptual learning results from interaction between bottom-up sensory signals and top-down reinforcement signals that can enhance learning probability. Reinforcement signals include reward, and alertness. In this schema, task-relevant learning occurs as a result of interaction between task-relevant features and reinforcement signals, 
and task-irrelevant learning between task-irrelevant features and reinforcement signals. Moreover, the model predicts conditions in which TIPL should occur, which is supported in the later study ${ }^{67}$.

\section{What changes occur in neural processing during the real- or near-real- time course of learning?}

There are at least two lines of time course training studies of perceptual learning. One focuses on short-term (up to a few weeks) and the other on long-term effects (months or years) of visual stimulus training on perceptual learning.

Research by Yotsumoto, Watanabe, and Sasaki on the effects of short-term training of an orientation discrimination task revealed two distinctive patterns of dynamic relations between task performance enhancement and neural activation changes measured via functional magnetic resonance imaging (fMRI) in the human brain ${ }^{24}$. It is known that a stimulus presented to a certain location in the visual field activates a specific local subregion of V1. During the initial few weeks of training, neural activation in the sub-region of V1 that corresponds to the trained visual field location (trained location) increased with task performance enhancement. However, after performance increase saturation occurred the neural activity increase disappeared, returning to the pre-training level. In contrast, increased performance on the perceptual task was maintained. This suggests that the initial (shortterm) stage of perceptual learning involves reorganization of a local neural network in V1 while learning is being acquired. Interestingly, after this early learning period, high performance is maintained in the newly reorganized/consolidated system without the need for further neural modification. Thus, it is important to take the length of training into account in the investigation of neural correlates of perceptual learning.

Research on determining whether perceptual learning is long-term or short-lived, revealed that task-relevant visual learning resulting from training on an orientation discrimination task was retained for 1.5 years after termination of the training task ${ }^{10}$. Also, task-irrelevant perceptual learning of a feature was found to last for at least 4 to 6 months after offset of exposure to the feature ${ }^{9}$. Thus, changes resulting from task-relevant and task-irrelevant exposure to visual stimuli do not represent mere training effects that disappear quickly upon training offset. Rather, they constitute long-term, persistent neural plasticity.

\section{What are the effects of feedback regarding correct or incorrect subject responses?}

Another interesting finding was that external feedback, such as an auditory signal specifying a subject's correct or incorrect responses is not necessary for perceptual learning ${ }^{23,61}$. The lack of feedback requirement also appeared to strongly support the hypothesis that perceptual learning occurs in the low-level, early visual processing system and follows mainly exposure-dependent perceptual processing rules that adjust the strength of neural connections according to the specific features of the observed stimulus.

The effects of providing subjects with feedback information regarding correct or incorrect responses on perceptual learning tasks have been studied extensively. This question is highly related to the brain algorithm(s), or theoretical internal calculations, involved in perceptual learning. Early studies in the 1980's indicated that task performance feedback is not required for perceptual learning to occur, suggesting that perceptual learning results from stimulusdriven internal processing. The lack of requirement of feedback led some researchers to suggest that perceptual learning follows an 'unsupervised learning' algorithm in which 
learning occurs based on feed-forward (bottom-up) processing independent of top-down processes (i.e. internal feedback signals).

Subsequently, Michael Herzog and Manfred Fahle conducted a systematic reinvestigation on the effects of feedback on perceptual learning ${ }^{68}$. While they found that response feedback presented on a trial-by-trial basis facilitates perceptual learning, they also found that a block feedback condition, in which correct performance feedback ratio was averaged across trials in a block, in this case, feedback was provided immediately after a set block of trials was completed, is as effective at facilitating trial-by-trial feedback learning.

Furthermore, Herzog and Fahle ${ }^{69}$ investigated whether feedback biases decision or encoding of stimuli. They trained subjects with a combination of correct and incorrect response feedback to a three dot Vernier (three vertically aligned dots) discrimination task. In this task, the subjects had to judge whether the central dot was shifted to the right or left relative to the top and bottom dots. The degree of the central dot shift was varied from small to large. When the central dot shifted slightly to the left side, the subject was provided with a feedback signal indicating the shift is right side. For example, assume that the dot shiftily slightly to the left. If the subject's response was "to the right", feedback indicated that the response was "correct" and if the response was "to the left", feedback indicated that the response was "incorrect". Other than the slight-shift-toward-left condition, the subject was administered a normal feedback signal. After exposure to these training conditions, subjects adjusted their decision criteria to respond according to the feedback. Interestingly, subjects for whom the criteria had once been set toward the incorrect feedback started making correct responses soon after they were provided only with correct feedback. Herzog and Fahle 69 concluded that feedback to a trained visual feature does not change the mechanisms related to sensitivity to the feature. Mechanisms through which feedback affects perceptual learning remain to be clarified by future research.

\section{Double training}

Recently, another important set of new findings in the literature on perceptual learning has been taking place: that is, so called double-training on transfer for position and other factors $32,70,71$. As described above, the feature specificity is often interpreted as strong involvement of the early visual area in perceptual learning. However, another interpretation may be possible. According to Mollon and Danilova ${ }^{72}$, feature specificity itself does not prove where the learning occurs; Learning occurs in a central site, which is beyond the early visual area, but at the same time brain needs to learn to attend a local location or orientation, which is expressed as feature specific. Xiao et al. (2008) tested this hypothesis by developing a new paradigm called double-training where conventional feature training is conducted at one location and additional training with an irrelevant feature at other location ${ }^{70}$. Intriguingly, they found that the additional location training enabled a complete transfer of the feature trained at the initial location to the second location, even though the feature was not trained at all at the second location. This finding challenges the interpretation of the feature specificity as the involvement of the early visual area in perceptual learning.

However, not all double-training enables complete transfer. More recently, Pilly et al showed lack of learning transfer even with the double-training paradigm ${ }^{73}$. They used a random dot motion stimulus and the contrast polarity specificity was dealt. The doubletraining paradigm may break the location specificity ${ }^{70}$, whereas it may not break the contrast polarity specificity. This controversy raises the question whether the underlying mechanism of perceptual learning is the same for any kinds of perceptual tasks. Thus, future 
studies should address the question whether a sole principle should explain all kinds of perceptual learning.

\section{Summary}

In summary, which stages of visual processing are involved in perceptual learning may depend on various conditions, including type of training task, time-course or duration of training, presence or absence of feedback and the training paradigm. In addition, as a whole, research suggests that perceptual learning may involve multiple stages from low-level perceptual processing in which primitive sensory elements are represented to higher decision-making neural units in which sensory representations are transformed to responses 24,31 . Future research should determine the interplay between the issues discussed here in resultant perceptual learning, as well as provide information concerning yet unexplored or identified contributing factors relevant to perceptual learning.

\section{References}

1. Morishita H, Hensch TK. Critical period revisited: impact on vision. Curr Opin Neurobiol. 2008; 18:101-107. [PubMed: 18534841]

2. Hubel DH, Wiesel TN. The period of susceptibility to the physiological effects of unilateral eye closure in kittens. J Physiol. 1970; 206:419-436. [PubMed: 5498493]

3. Wiesel TN, Hubel DH. Single-Cell Responses in Striate Cortex of Kittens Deprived of Vision in One Eye. J Neurophysiol. 1963; 26:1003-1017. [PubMed: 14084161]

4. Gibson EJ. Perceptual learning. Annu Rev Psychol. 1963; 14:29-56. [PubMed: 13947716]

5. Fahle, M.; Poggio, T. Perceptual learning. MIT Press; Cambridge, Mass: 2002.

6. De Valois KK. Spatial frequency adaptation can enhance contrast sensitivity. Vision Res. 1977; 17:1057-1065. [PubMed: 595415]

7. McKee SP, Westheimer G. Improvement in vernier acuity with practice. Percept Psychophys. 1978; 24:258-262. [PubMed: 704286]

8. Fiorentini A, Berardi N. Perceptual learning specific for orientation and spatial frequency. Nature. 1980; 287:43-44. [PubMed: 7412873]

9. Watanabe T, et al. Greater plasticity in lower-level than higher-level visual motion processing in a passive perceptual learning task. Nat Neurosci. 2002; 5:1003-1009. [PubMed: 12219093]

10. Karni A, Sagi D. The time course of learning a visual skill. Nature. 1993; 365:250-252. [PubMed: 8371779]

11. Polley DB, Steinberg EE, Merzenich MM. Perceptual learning directs auditory cortical map reorganization through top-down influences. J Neurosci. 2006; 26:4970-4982. [PubMed: 16672673]

12. Wilson DA, Fletcher ML, Sullivan RM. Acetylcholine and olfactory perceptual learning. Learn Mem. 2004; 11:28-34. [PubMed: 14747514]

13. Davis CM, et al. Discriminative stimulus properties of naloxone in Long-Evans rats: assessment with the conditioned taste aversion baseline of drug discrimination learning. Psychopharmacology (Berl). 2009; 203:421-429. [PubMed: 18594795]

14. Dinse HR, Ragert P, Pleger B, Schwenkreis P, Tegenthoff M. Pharmacological modulation of perceptual learning and associated cortical reorganization. Science. 2003; 301:91-94. [PubMed: 12843392]

15. Goldstone RL. Perceptual learning. Annu Rev Psychol. 1998; 49:585-612. [PubMed: 9496632]

16. Adini Y, Sagi D, Tsodyks M. Context-enabled learning in the human visual system. Nature. 2002; 415:790-793. [PubMed: 11845209]

17. Karni A, Sagi D. Where practice makes perfect in texture discrimination: evidence for primary visual cortex plasticity. Proc Natl Acad Sci U S A. 1991; 88:4966-4970. [PubMed: 2052578]

18. Ball K, Sekuler R. Direction-specific improvement in motion discrimination. Vision Res. 1987; 27:953-965. [PubMed: 3660656] 
19. Crist RE, Kapadia MK, Westheimer G, Gilbert CD. Perceptual learning of spatial localization: specificity for orientation, position, and context. J Neurophysiol. 1997; 78:2889-2894. [PubMed: 9405509]

20. Saarinen J, Levi DM. Perceptual learning in vernier acuity: what is learned? Vision Res. 1995; 35:519-527. [PubMed: 7900292]

21. Sagi D, Tanne D. Perceptual learning: learning to see. Curr Opin Neurobiol. 1994; 4:195-199. [PubMed: 8038576]

22. Fahle M, Edelman S. Long-term learning in vernier acuity: effects of stimulus orientation, range and of feedback. Vision Res. 1993; 33:397-412. [PubMed: 8447110]

23. Poggio T, Fahle M, Edelman S. Fast perceptual learning in visual hyperacuity. Science. 1992; 256:1018-1021. [PubMed: 1589770]

24. Yotsumoto Y, Watanabe T, Sasaki Y. Different dynamics of performance and brain activation in the time course of perceptual learning. Neuron. 2008; 57:827-833. [PubMed: 18367084]

25. Shiu LP, Pashler H. Improvement in line orientation discrimination is retinally local but dependent on cognitive set. Percept Psychophys. 1992; 52:582-588. [PubMed: 1437491]

26. Schoups AA, Vogels R, Qian N, Orban G. Practising orientation identification improves orientation coding in V1 neurons. PG - 549-53. Nature. 2001; 412

27. Nazir TA, O'Regan JK. Some results on translation invariance in the human visual system. Spat Vis. 1990; 5:81-100. [PubMed: 2090197]

28. Mednick SC, Arman AC, Boynton GM. The time course and specificity of perceptual deterioration. Proc Natl Acad Sci U S A. 2005; 102:3881-3885. [PubMed: 15731350]

29. Yu C, Klein SA, Levi DM. Perceptual learning in contrast discrimination and the (minimal) role of context. J Vis. 2004; 4:169-182. [PubMed: 15086307]

30. Jeter PE, Dosher BA, Liu SH, Lu ZL. Specificity of perceptual learning increases with increased training. Vision Res. 2010; 50:1928-1940. [PubMed: 20624413]

31. Ahissar M, Hochstein S. Task difficulty and the specificity of perceptual learning. Nature. 1997; 387:401-406. [PubMed: 9163425]

32. Zhang JY, et al. Stimulus coding rules for perceptual learning. PLoS Biol. 2008; 6:e197. [PubMed: 18707195]

33. Schoups AA, Vogels R, Orban GA. Human perceptual learning in identifying the oblique orientation: retinotopy, orientation specificity and monocularity. J Physiol. 1995; 483 ( Pt 3):797810. [PubMed: 7776259]

34. Ball K, Sekuler R. Adaptive processing of visual motion. J Exp Psychol Hum Percept Perform. 1981; 7:780-794. [PubMed: 6457092]

35. Koyama S, Harner A, Watanabe T. Task-dependent changes of the psychophysical motion-tuning functions in the course of perceptual learning. Perception. 2004; 33:1139-1147. [PubMed: 15560512]

36. Vaina LM, Belliveau JW, des Roziers EB, Zeffiro TA. Neural systems underlying learning and representation of global motion. Proc Natl Acad Sci U S A. 1998; 95:12657-12662. [PubMed: 9770542]

37. Matthews N, Liu Z, Geesaman BJ, Qian N. Perceptual learning on orientation and direction discrimination. Vision Res. 1999; 39:3692-3701. [PubMed: 10746139]

38. Seitz AR, Kim D, Watanabe T. Rewards evoke learning of unconsciously processed visual stimuli in adult humans. Neuron. 2009; 61:700-707. [PubMed: 19285467]

39. Aslin C, Blake R, Chun MM. Perceptual learning of temporal structure. Vision Res. 2002; 42:3019-3030. [PubMed: 12480072]

40. Lu ZL, Chu W, Dosher BA, Lee S. Independent perceptual learning in monocular and binocular motion systems. Proc Natl Acad Sci U S A. 2005; 102:5624-5629. [PubMed: 15802469]

41. Schoups AA, Orban GA. Interocular transfer in perceptual learning of a pop-out discrimination task. Proc Natl Acad Sci U S A. 1996; 93:7358-7362. [PubMed: 8692998]

42. Maunsell JH, Newsome WT. Visual processing in monkey extrastriate cortex. Annu Rev Neurosci. 1987; 10:363-401. [PubMed: 3105414] 
43. Albright TD. Direction and orientation selectivity of neurons in visual area MT of the macaque. J Neurophysiol. 1984; 52:1106-1130. [PubMed: 6520628]

44. Sasaki Y, Nanez JE, Watanabe T. Advances in visual perceptual learning and plasticity. Nat Rev Neurosci. 2010; 11:53-60. [PubMed: 19953104]

45. Lu ZL, Hua T, Huang CB, Zhou Y, Dosher BA. Visual perceptual learning. Neurobiol Learn Mem. 2011; 95:145-151. [PubMed: 20870024]

46. Sagi D. Perceptual learning in Vision Research. Vision Res. 2011; 51:1552-1566. [PubMed: 20974167]

47. Schoups AA, Vogels R, Qian N, Orban G. Practising orientation identification improves orientation coding in V1 neurons. Nature. 2001; 412:549-553. [PubMed: 11484056]

48. Crist RE, Li W, Gilbert CD. Learning to see: experience and attention in primary visual cortex. Nat Neurosci. 2001; 4:519-525. [PubMed: 11319561]

49. Li W, Gilbert CD. Global contour saliency and local colinear interactions. J Neurophysiol. 2002; 88:2846-2856. [PubMed: 12424317]

50. Li W, Piech V, Gilbert CD. Perceptual learning and top-down influences in primary visual cortex. Nat Neurosci. 2004; 7:651-657. [PubMed: 15156149]

51. Hua T, et al. Perceptual learning improves contrast sensitivity of V1 neurons in cats. Curr Biol. 2010; 20:887-894. [PubMed: 20451388]

52. Schwartz S, Maquet P, Frith C. Neural correlates of perceptual learning: a functional MRI study of visual texture discrimination. Proc Natl Acad Sci U S A. 2002; 99:17137-17142. [PubMed: 12446842]

53. Furmanski CS, Schluppeck D, Engel SA. Learning strengthens the response of primary visual cortex to simple patterns. Curr Biol. 2004; 14:573-578. [PubMed: 15062097]

54. Petrov AA, Dosher BA, Lu ZL. The dynamics of perceptual learning: an incremental reweighting model. Psychol Rev. 2005; 112:715-743. [PubMed: 16262466]

55. Yang T, Maunsell JH. The effect of perceptual learning on neuronal responses in monkey visual area V4. J Neurosci. 2004; 24:1617-1626. [PubMed: 14973244]

56. Raiguel S, Vogels R, Mysore SG, Orban GA. Learning to see the difference specifically alters the most informative V4 neurons. J Neurosci. 2006; 26:6589-6602. [PubMed: 16775147]

57. Law CT, Gold JI. Neural correlates of perceptual learning in a sensory-motor, but not a sensory, cortical area. Nat Neurosci. 2008; 11:505-513. [PubMed: 18327253]

58. Gold JI, Shadlen MN. Representation of a perceptual decision in developing oculomotor commands. Nature. 2000; 404:390-394. [PubMed: 10746726]

59. Shibata K, Watanabe T, Sasaki Y, Kawato M. Perceptual learning incepted by decoded fMRI neurofeedback without stimulus presentation. Science. 2011; 334:1413-1415. [PubMed: 22158821]

60. Ahissar M, Hochstein S. Attentional control of early perceptual learning. Proc Natl Acad Sci U S A. 1993; 90:5718-5722. [PubMed: 8516322]

61. Fahle M, Edelman S, Poggio T. Fast perceptual learning in hyperacuity. Vision Res. 1995; 35:3003-3013. [PubMed: 8533337]

62. Weiss Y, Edelman S, Fahle M. Models of perceptual learning in vernier acuity. Neural Computation. 1993; 5:695-718.

63. Moses Y, Schechtman G, Ullman S. Self-calibrated collineary detector. Biological Cybernetics. 1990; 63:463-475.

64. Polat U, Sagi D. Spatial interactions in human vision: from near to far via experience-dependent cascades of connections. Proc Natl Acad Sci U S A. 1994; 91:1206-1209. [PubMed: 8108388]

65. Watanabe T, Nanez JE, Sasaki Y. Perceptual learning without perception. Nature. 2001; 413:844848. [PubMed: 11677607]

66. Seitz A, Watanabe T. A unified model for perceptual learning. Trends Cogn Sci. 2005; 9:329-334. [PubMed: 15955722]

67. Tsushima Y, Seitz A, Watanabe T. Task-irrelevant learning occurs only when the irrelevant feature is weak. Current Biol. 2008 in press. 
68. Herzog MH, Fahle M. The role of feedback in learning a vernier discrimination task. Vision Res. 1997; 37:2133-2141. [PubMed: 9327060]

69. Herzog MH, Fahle M. Effects of biased feedback on learning and deciding in a vernier discrimination task. Vision Res. 1999; 39:4232-4243. [PubMed: 10755160]

70. Xiao LQ, et al. Complete transfer of perceptual learning across retinal locations enabled by double training. Curr Biol. 2008; 18:1922-1926. [PubMed: 19062277]

71. Wang R, Zhang JY, Klein SA, Levi DM, Yu C. Task relevancy and demand modulate doubletraining enabled transfer of perceptual learning. Vision Res. 2011

72. Mollon JD, Danilova MV. Three remarks on perceptual learning. Spat Vis. 1996; 10:51-58. [PubMed: 8817771]

73. Pilly PK, Grossberg S, Seitz AR. Low-level sensory plasticity during task-irrelevant perceptual learning: evidence from conventional and double training procedures. Vision Res. 2010; 50:424432. [PubMed: 19800358] 